PHYSICAL REVIEW D 95, 039901(E) (2017)

\title{
Erratum: Rare decays of $B$ mesons via on-shell sterile neutrinos [Phys. Rev. D 94, 053001 (2016)]
}

\author{
Gorazd Cvetić and C. S. Kim \\ (Received 26 January 2017; published 9 February 2017)
}

DOI: 10.1103/PhysRevD.95.039901

In Fig. 4(b), the lower curve [for $\bar{\Gamma}\left(\bar{B}^{0} \rightarrow D^{*+} \tau^{-} \bar{N}\right]$ was not correct due to an inadvertent use of an obsolete curve in the plotting program. Below we present this figure with the correct curve. Equations (C20b) and (19), on which the curves in Fig. 4(b) are based, are correct. All the figures in the paper which involve reactions with $D^{*}$ in the final state and use these results are correct.

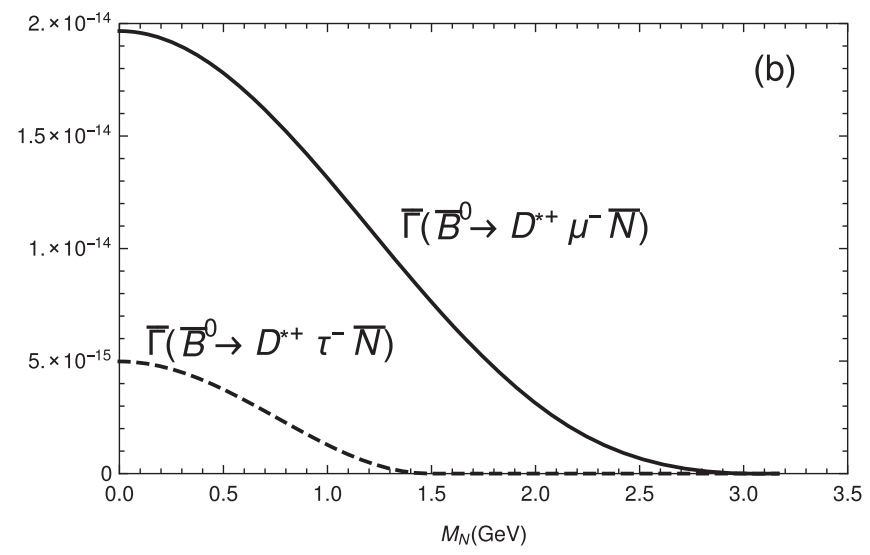

FIG. 4. The canonical decay widths of $B$ meson into $D^{*} \ell N$, as a function of the mass of $N$ neutrino. The specific cases of $\ell=\mu, \tau$ are presented. The decay widths are in units of GeV.

In Appendix $\mathrm{C}$, there is a typo in Eq. (C15a). On the right-hand side the fraction should contain the factor $2 M_{B}$ in the numerator. The correct form is

$$
\bar{H}_{ \pm 1}=\left(M_{B}+M_{D^{*}}\right) A_{1}\left(q^{2}\right) \mp V\left(q^{2}\right) \frac{|\vec{q}| 2 M_{B}}{\left(M_{B}+M_{D^{*}}\right)} .
$$

The other formulas following this equation in Appendix $\mathrm{C}$ are correct, including expressions $(\mathrm{C} 17)-(\mathrm{C} 20)$.

Equation (C2) in Appendix $\mathrm{C}$ has a minor typo. $\varepsilon^{\nu \nu \alpha \beta}$ should be written as $\varepsilon^{\mu \nu \alpha \beta}$. 